\title{
Global Potential Energy Surfaces with Correct Permutation Symmetry by Multi-Configuration Molecular Mechanics
}

\author{
Oksana Tishchenko* and Donald G. Truhlar* \\ Chemistry Department and Supercomputing Institute, University of Minnesota, \\ Minneapolis, MN 55455-0431
}

Received date: October 252006

\begin{abstract}
In the framework of the previously developed multi-configuration molecular mechanics (MCMM) method, we present a new algorithm for constructing global potential energy surfaces that are invariant with respect to the exchange of identical nuclei. We illustrate the new algorithm by its application to the $\mathrm{HOH}^{\prime \prime}+\mathrm{H}^{\prime} \rightarrow \mathrm{OH}+\mathrm{H}^{\prime} \mathrm{H}^{\prime \prime}, \mathrm{OH}^{\prime}+\mathrm{HH}^{\prime \prime}$, $\mathrm{OH}^{\prime \prime}+\mathrm{HH}^{\prime}, \mathrm{HOH}^{\prime}+\mathrm{H}^{\prime \prime}$, and $\mathrm{H}^{\prime \prime} \mathrm{OH}^{\prime}+\mathrm{H}$ reactions. As part of the MCMM methodology, the new scheme can be used to generate multidimensional global PESs for both small and large systems where a few reaction pathways need to be treated as symmetrically equivalent.
\end{abstract}

\footnotetext{
*Corresponding author email: truhlar@umn.edu (D.G.T.) and oksana@t1.chem.umn.edu (O.T.)
} 


\section{Introduction}

Multi-configuration molecular mechanics provides an efficient and systematic scheme for extending molecular mechanics ${ }^{1-23}$ potentials to allow the fitting of potential energy surfaces for reactive systems. ${ }^{24-29}$ This is accomplished by using molecular mechanics to model the diagonal matrix elements ${ }^{30}$ of the electronic Hamiltonian in a diabatic basis and using electronic structure calculations of the adiabatic electronic ground state to obtain the off-diagonal couplings. In applications so far, we have considered fitting a potential energy surface in a single reaction swath, which consists of those geometries in the valley around a single minimum energy path plus the region on the concave side of that reaction path that is necessary for a semiclassical treatment of large-curvature tunneling. ${ }^{31-36}$ This requires input data only in the reaction swath itself and allows the calculation of accurate rate constants by generalized transition state theory and in particular by variational transition state theory with multidimensional tunneling. ${ }^{37-42}$ To obtain potential energy surfaces more suitable for general dynamics calculations, such as trajectory calculations, ${ }^{43-46}$ one can add additional data far from the minimum-energy reaction paths, for example, by the "Grow" algorithm of Collins. $^{47}$

In some cases, it is desired to model more than one reaction path. Consider for example the reaction

$$
\mathrm{H}_{2} \mathrm{O}+\mathrm{H} \rightarrow \mathrm{OH}+\mathrm{H}_{2}
$$

For calculating thermal rate constants by generalized transition state theory, there is no loss of accuracy in labeling the atoms:

$$
H_{a} O H_{b}+H_{c} \rightarrow O H_{a}+H_{b} H_{c}
$$

and treating the path connecting these labeled asymptotes as the only low-energy one because reactive flux via the reaction swath associated with the path leading to $\mathrm{OH}_{b}+\mathrm{H}_{a} \mathrm{H}_{c}$ can be included by a symmetry factor, i.e., by multiplying the rate constant for path (2) by two. 
However, trajectory calculations, which might be carried out to study high-energy processes or state-selective properties can access geometries where $\mathrm{H}_{a}$ and $\mathrm{H}_{b}$ are equally distant from $\mathrm{H}_{c}$. The fitted potential energy surface should be invariant to permutations of identical nuclei when geometries connecting specific reaction swaths are energetically accessible. Huang et al. ${ }^{48}$ have recently presented a permutationally invariant fitting basis for enforcing identical particle symmetry in fits of potential energy surfaces. Collins et al. ${ }^{47}$ have used an alternative strategy where all permutationally equivalent geometries are included in the data to be fit. A key element of the present approach is that we use symmetrized diabatic states. This allows us to include the permutation symmetry of selected identical nuclei without increasing the order of the electronic Hamiltonian matrix.

In the present article, we present a general method for enforcing permutation symmetry in MCMM calculations, and we demonstrate it by making a global potential energy surface for the model reaction $\mathrm{H}_{2} \mathrm{O}+\mathrm{H}$. We illustrate this surface by showing the geometric characteristics of the fit at various molecular geometries. The method is designed for cases where it is sufficient to treat a few exchangeable atoms (typically a few hydrogens) in a symmetrical way and not for treating thousands of hydrogen atoms (e.g. in a protein) symmetrically, which is seldom necessary.

\section{Theory}

In MCMM, the reactive system is defined using $n$ valence bond (or diabatic) configurations that correspond to reactants, products, and (possibly) stable or metastable intermediates. Each valence bond configuration corresponds to a definite arrangement of bonds. In the present work, we only consider two configurations: $n=1$, corresponding to the reactant arrangement of bonds, and $n=2$, corresponding to the product. Near potential energy minima, the diabatic configurations are assumed to be well described by available molecular mechanics potentials $V_{n n}(\mathbf{q})$, where $\mathbf{q}$ denotes a set of redundant or nonredundant internal 
valence coordinates (bond stretches, bond angle bends, and torsions). At an arbitrary geometry, the potential energy $V$ can be expressed in terms of these $V_{n n}$ by solving the secular equation:

$$
\left|\begin{array}{cc}
V_{11}-V & V_{12} \\
V_{12} & V_{22}-V
\end{array}\right|=0
$$

where $V_{12}$ is the resonance integral (or diabatic coupling), and $V$ is the lowest eigenvalue of the matrix $\mathbf{V}$. If analytic gradients and Hessians with respect to nuclear coordinates are available for both $V_{n n}$ and $V_{n n^{\prime}}$, then the analytic gradient and Hessian of $V$ are readily available by applying the chain rule to the analytic solution of eq 3 .

We consider a reactive system with $m$ identical nuclei (i.e., nuclei with the same atomic number, e.g., hydrogen atoms). First, we define two sets of permutation operators. The first set is the group $\left\{\mathbf{P}^{(i)}(i=1, \ldots m !)\right\}$ of nuclear permutation operators that interchange Cartesian coordinates $\mathbf{x}^{(k)}$ of identical nuclei and generate the permutationally equivalent geometries $\mathbf{x}^{(k, i)}$; this is the symmetric group ${ }^{49,50}$ of degree $m$ and order $m$ ! and corresponds to the complete nuclear permutation $(\mathrm{CNP})$ group $^{51}$ of a molecule. In the case of a system with three hydrogen atoms such as reaction 1 of $\mathrm{H}_{2} \mathrm{O}$ with $\mathrm{H}$, there are $m !=6$ permutationally equivalent molecular geometries and the group $\mathbf{P}^{(i)}$ has the following elements: the identity operator, three transposition operators, and two cyclic permutation operators: $\{E$, (12), (23), (13), (123), (132) $\}.{ }^{49,51}$ The second set, $\left\{\mathbf{P}^{M M(j)}(j=1, \ldots m !)\right\}$, consists of the operators that operate on the molecular mechanics atom types and connectivity patterns at identical nuclei centers while leaving their Cartesian coordinates unchanged. The result of the application of each of the $\mathbf{P}^{M M(i)}$ to a valence bond structure $n$ in which the atoms are labeled (e.g., $H_{a} O H_{b}+H_{c}$ ) is a change in the valence bond connectivity pattern of the nuclei so that $j=1, \ldots m$ ! generates all possible connectivity patterns with the same numbers and kinds of bonds (e.g., $H_{a} O H_{c}+H_{b}$ ). There is a one-to-one correspondence between the elements of the group $\mathbf{P}^{(i)}$ and applying the elements of the set $\mathbf{P}^{M M(j)}$, and this correspondence may be used to assign the labels $j$. 
Note that the permutations $\mathbf{P}^{M M(j)}$ usually change the energy of the system. For example, applying $\mathbf{P}^{M M(2)}$ to the labeled asymptotic form on the left side of eq 2 results in the same-energy structure $\left(H_{b} O H_{a}+H_{c}\right)$, whereas applying $\mathbf{P}^{M M(3)}$ to the same structure yields $H_{a} O H_{c}+H_{b}$ which would generally have higher MM energy unless the $O H_{b}$ and $O H_{c}$ distances are the same. Let $\sigma_{n}$, which is called the symmetry factor, be the number of times the lowest-energy MM configuration occurs among the $m$ ! symmetrically equivalent $\mathrm{MM}$ configurations at a general geometry. Note that there may be high-symmetry geometries where the lowest-energy MM configurations occur more than $\sigma_{n}$ times, but $\sigma_{n}$ is independent of geometry and corresponds to a general geometry. For example, for studying reaction (1), $n$ $=1$ is the $\mathrm{H} \cdots \mathrm{H}_{2} \mathrm{O}$ configuration, and $n=2$ is the $\mathrm{OH} \cdots \mathrm{H}_{2}$ configuration, and each has $\sigma_{n}=2$, although the lowest-energy configuration occurs 6 times for $C_{3 v}$ and $D_{3 h}$ geometries.

The requirement that $V$ be invariant with respect to the permutation of identical nuclei suggests that both the diagonal and off-diagonal matrix elements, $V_{n n}$ and $V_{n n^{\prime}}$, need to be invariant under such permutations. In MCMM, the diagonal matrix elements are expressed in terms of the pre-determined analytical MM functions, and the of-diagonal elements $V_{n n^{\prime}}$ are obtained via Shepard interpolation ${ }^{47,52-57}$ using accurate (quantum mechanical (QM)) data at a selected number of molecular geometries. In the interpolation, the data are weighted by a weight function so that data at nearby points are weighted more heavily than those at distant points. The resonance integrals $V_{n n^{\prime}}$ depend on both MM and QM energies and derivatives (gradients and Hessians). We will present the algorithm for symmetrizing both $V_{n n}$ and $V_{n n^{\prime}}$ in detail below, after a paragraph about the various internal coordinates that are used.

Internal coordinates will appear in the algorithm in three different contexts, and these sets need not necessarily be the same; in fact they ordinarily are not the same. In particular, there are (a) $2 m$ ! sets of internal coordinates $\mathbf{q}$ used to evaluate MM energies and their derivatives: $m$ ! sets for valence bond configuration $n=1$ and another $m$ ! sets for valence 
bond configuration $n=2$, (b) a set of internal coordinates $\mathbf{r}$ used in Shepard interpolation, and (c) a set of internal coordinates $\mathbf{s}$ used to calculate the weight function. The number of coordinates in set $\mathbf{r}$ is greater than or equal to $3 N_{A}-6$, where $N_{A}$ is the number of atoms in the system, and the number of coordinates in set $\mathbf{s}$ is arbitrary and is called $\Gamma$. The compositions of the sets $\mathbf{q}$ are completely determined by the molecular mechanics method (based on the valence bond connectivity patterns), but sets $\mathbf{r}$ and $\mathbf{s}$ are introduced in MCMM. It is worthwhile to explicitly mention that the coordinates $\mathbf{q}$ depend on $i$. Thus, for example, for $i=1$ and $n=2$, we have the $\mathrm{O}-\mathrm{H}_{a}$ and $\mathrm{H}_{b}-\mathrm{H}_{c}$ bond distances, and we have $\mathrm{O}-\mathrm{H}_{b}, \mathrm{O}-\mathrm{H}_{c}$, $\mathrm{H}_{a}-\mathrm{H}_{b}$, and $\mathrm{H}_{a}-\mathrm{H}_{c}$ as nonbonded coordinates. In contrast for $i=2$ we have $\mathrm{O}-\mathrm{H}_{b}$ and $\mathrm{H}_{a}-\mathrm{H}_{c}$ as bond distances and $\mathrm{O}-\mathrm{H}_{a}, \mathrm{O}-\mathrm{H}_{c}, \mathrm{H}_{b}-\mathrm{H}_{a}$, and $\mathrm{H}_{b}-\mathrm{H}_{c}$ as nonbonded coordinates. This is not reflected in the notation because it would be cumbersome. For symmetrized calculations, both sets (set b called $\mathbf{r}$ and set c called $\mathbf{s}$ ) should include all permutationally equivalent coordinates that involve the identical nuclei that need to be treated as permutationally equivalent. As described in the original MCMM paper, ${ }^{24}$ Shepard interpolation is carried out in internal coordinates rather than in Cartesians in order circumvent the problem of choosing a consistent orientation of Cartesian coordinates at the various interpolation nodes. In the symmetrized application presented below, we use all (six) internuclear distances (and no bond angles or torsions) for this Shepard interpolation step. For evaluating the weight function $W$, which is explained below, we would in general use the internuclear distances that undergo significant changes during the reaction of interest and, in addition, all permutationally equivalent distances. In the application presented below, we used the set of all internuclear distances for the weights.

The procedure for constructing a PES that is invariant with respect to the exchange of identical nuclei using MCMM involves the following steps:

(i) Read electronic structure information (accurate energies $V^{(k)}$, gradients $\mathbf{G}^{(k)}$, and Hessians $\mathbf{F}^{(k)}$ ) in Cartesian coordinates for $k=1,2, \ldots N$ training geometries $\mathbf{x}^{(k)}$, and for 
each of these data points generate $m$ ! symmetrically equivalent data sets: $\left\{\mathbf{x}^{(k, i)}, \mathbf{G}^{(k, i)}\right.$, $\left.\mathbf{F}^{(k, i)}\right\}$, where

$$
\begin{gathered}
\mathbf{x}^{(k, i)}=\mathbf{P}^{(i)} \mathbf{x}^{(k)} \\
\mathbf{G}^{(k, i)} \equiv \frac{\partial}{\partial \mathbf{x}} V=\mathbf{P}^{(i)} \mathbf{G}^{(k)} \\
\mathbf{F}^{(k, i)} \equiv \frac{\partial^{2}}{\partial \mathbf{x}^{2}} V=\mathbf{P}^{(i)} \mathbf{F}^{(k)} \mathbf{P}^{(i)}
\end{gathered}
$$

Notice that $\mathbf{x}^{(k, 1)}=\mathbf{x}^{(k)}, \mathbf{G}^{(k, 1)}=\mathbf{G}^{(k)}, \mathbf{F}^{(k, 1)}=\mathbf{F}^{(k)}$, and $\mathbf{P}^{(1)}$ corresponds to $E, \mathbf{P}^{(2)}$ corresponds to (12), etc. Notice also that eq 5 corresponds to permuting rows in the gradient vector, and eq 6 corresponds to permuting rows and columns in the Hessian matrix. The potential energy is a scalar and independent of $i$; we can call the accurate energy $V^{(k)}, V^{(k, 1)}$, or $V^{(k, i)}$.

(ii) Define a set of $m$ ! MM energies, gradients, and Hessians at point $(k)$ by:

$$
\begin{gathered}
V_{\mathrm{MM}, n}^{(k, j)} \equiv V_{n n}^{(j)}\left(\mathbf{x}^{(k)}\right) \\
\left.\mathbf{G}_{\mathrm{MM}, n}^{(k, j)} \equiv \frac{\partial}{\partial \mathbf{x}} V_{n n}^{(j)}\right|_{\mathbf{x}=\mathbf{x}^{(k)}}
\end{gathered}
$$

and

$$
\left.\mathbf{F}_{\mathrm{MM}, n}^{(k, j)} \equiv \frac{\partial^{2}}{\partial \mathbf{x}^{2}} V_{n n}^{(j)}\right|_{\mathbf{x}=\mathbf{x}^{(k)}}
$$

for $n=1,2 ; k=1,2, \ldots, N ; j=1,2, \ldots, m$ !. This set corresponds to the $m$ ! different MM connectivity patterns generated by applying each $\mathbf{P}^{M M(j)}$ to a valence bond configuration $n$ at each geometry $\left(\mathbf{x}^{(k, 1)}\right)$. The MM energy, gradient, and Hessian on the right sides of eqs 7, 8 , and 9, respectively, are evaluated in the present article using the MM3 force field ${ }^{5-7}$ modified as described in a previous paper, ${ }^{29}$ although the algorithm is general and can also be applied with other force fields, e.g., with CHARMM. ${ }^{18,21}$ In our work the Cartesian derivatives of eqs 8 and 9 are evaluated from the molecular mechanics force fields by the TINKER program. ${ }^{58}$ (TINKER evaluates the derivatives in the internal coordinate set $\mathbf{q}$ and then transforms them to Cartesian coordinates.) 
(iii) Define a symmetrized MM potential and its gradient and Hessian at point $k$ (where a tilde denotes a symmetrization) by:

$$
\tilde{V}_{n}^{(k)}=-\Delta \ln \left(\frac{1}{\sigma_{n}} \sum_{j}^{m !} e^{-V_{\mathrm{MM}, n}^{(k, j)} / \Delta}\right),
$$

where $\Delta$ is a parameter;

$$
\tilde{\mathbf{G}}_{n}^{(k)} \equiv \frac{\partial}{\partial \mathbf{x}} \tilde{V}_{n}^{(k)}=\frac{\sum_{j}^{m !} \mathbf{G}_{\mathrm{MM}, n}^{(k, j)} e^{-V_{\mathrm{MM}, n}^{(k, j)} / \Delta}}{\sum_{j}^{m !} e^{-V_{\mathrm{MM}, n}^{(k, j)} / \Delta}}
$$

and

$$
\tilde{\mathbf{F}}_{n}^{(k)} \equiv \frac{\partial^{2}}{\partial \mathbf{x}^{2}} \tilde{V}_{n}^{(k)}=\frac{\sum_{j}^{m !}\left(\mathbf{F}_{\mathrm{MM}, n}^{(k, j)}-(1 / \Delta) \mathbf{G}_{\mathrm{MM}, n}^{(k, j)} \mathbf{G}_{\mathrm{MM}, n}^{(k, j) \mathbf{T}}\right) e^{-V_{n}^{(k, j)} / \Delta}}{\sum_{j}^{m !} e^{-V_{\mathrm{MM}, n}^{(k, j)} / \Delta}}+(1 / \Delta) \tilde{\mathbf{G}}_{n}^{(k)} \tilde{\mathbf{G}}_{n}^{(k) \mathrm{\top}} \sum_{j}^{m !} e^{-V_{\mathrm{MM}, n}^{(k, j)} / \Delta} .
$$

Notice that the symmetrized MM potential is dominated by the $\sigma_{n}$ lowest-energy MM configurations among the $m$ ! permutations of the labels on the identical atoms. The parameter $\Delta$ controls the rate of switching between different dominant configurations in regions that separate the low-energy regions corresponding to the differently permuted coordinates.

We now have the accurate potential, its gradient, and Hessian (eqs 4-6), and the symmetrized MM potential, gradient, and Hessian (eqs 10-12) at each of the $N$ training points. Note that $V^{(k)}, \tilde{V}_{n}^{(k)}, \tilde{\mathbf{G}}_{n}^{(k)}$, and $\tilde{\mathbf{F}}_{n}^{(k)}$ are independent of $i$, but $\mathbf{G}^{(k, i)}$ and $\mathbf{F}^{(k, i)}$ depend on $i$. (Because of the dependence of these quantities on $i$, the Shepard interpolation must involve $m ! N$ terms, rather than $N$ terms, as in the unsymmetrical case.)

(iv) Generate $m$ ! values of $\tilde{\mathbf{G}}_{n}^{(k, i)}$ and $\tilde{\mathbf{F}}_{n}^{(k, i)}$ from each $\tilde{\mathbf{G}}^{(k)}$, and $\tilde{\mathbf{F}}^{(k)}$ by applying $\mathbf{P}^{(i)}$, as in step (i). 
(v) Then we transform $\mathbf{G}^{(k, i)}, \mathbf{F}^{(k, i)}, \tilde{\mathbf{G}}_{n}^{(k, i)}$, and $\tilde{\mathbf{F}}_{n}^{(k, i)}$ to the set of internal coordinates $\mathbf{r}$ by the Wilson B matrix and C tensor, as described elsewhere. ${ }^{24,59,60}$ This yields:

$$
\begin{aligned}
\mathbf{g}^{(k, i)} & \left.\equiv \frac{\partial}{\partial \mathbf{r}} V\right|_{\mathbf{r}=\mathbf{r}\left(\mathbf{x}^{(k, i)}\right)} \\
\mathbf{f}^{(k, i)} & \left.\equiv \frac{\partial^{2}}{\partial \mathbf{r}^{2}} V\right|_{\mathbf{r}=\mathbf{r}\left(\mathbf{x}^{(k, i)}\right)} \\
\tilde{\mathbf{g}}_{n}^{(k, i)} & \left.\equiv \frac{\partial}{\partial \mathbf{r}} \tilde{V}_{n}\right|_{\mathbf{r}=\mathbf{r}\left(\mathbf{x}^{(k, i)}\right)} \\
\tilde{\mathbf{f}}_{n}^{(k, i)} & \left.\equiv \frac{\partial^{2}}{\partial \mathbf{r}^{2}} \tilde{V}_{n}\right|_{\mathbf{r}=\mathbf{r}\left(\mathbf{x}^{(k, i)}\right)}
\end{aligned}
$$

Throughout this paper, we will use capital $\mathbf{G}$ and $\mathbf{F}$ to denote the gradients and Hessians with respect to Cartesian coordinates, and lower case $\mathbf{g}$ and $\mathbf{f}$ to denote the corresponding derivatives with respect to internal coordinates.

(vi) Next we define two Taylor series for each data point $(k, i)$ by:

$$
V(\mathbf{r} ; k) \approx V^{(k, i)}+\mathbf{g}^{(k, i) \top} \Delta \mathbf{r}+\frac{1}{2} \Delta \mathbf{r}^{(k, i) \top} \mathbf{f}^{(k, i)} \Delta \mathbf{r}^{(k, i)},
$$

and

$$
\tilde{V}_{n}(\mathbf{r} ; k) \approx \tilde{V}_{n}^{(k, i)}+\tilde{\mathbf{g}}_{n}^{(k, i) \top} \Delta \mathbf{r}+\frac{1}{2} \Delta \mathbf{r}^{(k, i) \top} \tilde{\mathbf{f}}_{n}^{(k, i)} \Delta \mathbf{r}^{(k, i)},
$$

where $V(\mathbf{r} ; k), \mathbf{g}^{(k, i)}, \mathbf{f}^{(k, i)}, \tilde{V}(\mathbf{r} ; k), \tilde{\mathbf{g}}_{n}^{(k, i)}$ and $\tilde{\mathbf{f}}_{n}^{(k, i)}$ are the QM potential, gradient and Hessian, and the symmetrized MM potential, gradient and Hessian in the internal coordinates, and

$$
\Delta \mathbf{r}^{(k, i)}=\mathbf{r}(\mathbf{x})-\mathbf{r}\left(\mathbf{x}^{(k, i)}\right),
$$

where $\mathbf{r}\left(\mathbf{x}^{k, i}\right)$ is the value of $\mathbf{r}$ at point $(k, i)$. The Taylor series coefficients, denoted as $D, \mathbf{b}$, and $\mathbf{C}$, needed for the $m ! N$-term interpolation are then calculated by substituting $V^{(k)}, \tilde{V}_{n}^{(k)}$, $\mathbf{g}^{(k, i)}, \mathbf{f}^{(k, i)}, \tilde{\mathbf{g}}_{n}^{(k, i)}$, and $\tilde{\mathbf{f}}_{n}^{(k, i)}$ (for $\left.n=1,2\right)$ into eqs 20-23 of ref 24, with the only difference being that $(k)$ in these equations is now replaced by $(k, i)$ :

$$
D^{(k, i)}=\left(\tilde{V}_{1}^{(k, i)}-V^{(k, i)}\right)\left(\tilde{V}_{2}^{(k, i)}-V^{(k, i)}\right)
$$




$$
\begin{gathered}
\mathbf{b}^{(k, i)}=\frac{\tilde{\mathbf{g}}_{1}^{(k, i)}-\mathbf{g}^{(k, i)}}{\tilde{V}_{1}^{(k, i)}-V^{(k, i)}}+\frac{\tilde{\mathbf{g}}_{2}^{(k, i)}-\mathbf{g}^{(k, i)}}{\tilde{V}_{2}^{(k, i)}-V^{(k, i)}} \\
\mathbf{C}^{(k, i)}=\left(1 / D^{(k, i)}\right)\left[\left(\tilde{\mathbf{g}}_{1}^{(k, i)}-\mathbf{g}^{(k, i)}\right)\left(\tilde{\mathbf{g}}_{2}^{(k, i)}-\mathbf{g}^{(k, i)}\right)^{\top}+\right. \\
\left.\left(\tilde{\mathbf{g}}_{2}^{(k, i)}-\mathbf{g}^{(k, i)}\right)\left(\tilde{\mathbf{g}}_{1}^{(k, i)}-\mathbf{g}^{(k, i)}\right)^{\top}\right]+\frac{\tilde{\mathbf{f}}_{1}^{(k, i)}-\mathbf{f}^{(k, i)}}{\tilde{V}_{1}^{(k, i)}-V^{(k, i)}}+\frac{\tilde{\mathbf{f}}_{2}^{(k, i)}-\mathbf{f}^{(k, i)}}{\tilde{V}_{2}^{(k, i)}-V^{(k, i)}}
\end{gathered}
$$

(vii) Then eq 18 (or equivalently eq 13 ) of ref 24 , with $(k, i)$ replacing $(k)$ gives the Taylor series of $\left(V_{12}\right)^{2}$ for each $(k, i)$ at an arbitrary geometry $\mathbf{r}=\mathbf{r}(\mathbf{x})$ along with its gradient and Hessian in the internal coordinates $\mathbf{r}$ :

$$
\left[V_{12}(\mathbf{r}, k, i)\right]^{2}=D^{(k, i)}\left(1+\mathbf{b}^{(k, i) \top} \Delta \mathbf{r}^{(k, i)}+\frac{1}{2} \Delta \mathbf{r}^{(k, i) \top} \mathbf{C}^{(k, i)} \Delta \mathbf{r}^{(k, i)}\right)
$$

This step uses the Taylor series reversion of Chang and Miller. ${ }^{61}$ (Notice that there is a typo in eq 13 of ref 24 , namely that all $\Delta \mathbf{q}$ in that equation should have been $\Delta \mathbf{q}^{(k)}$. Furthermore the internal coordinates used in that equation are the set called $\mathbf{r}$ in the present work. Therefore, $\Delta \mathbf{q}^{(k)}$ has now become $\Delta \mathbf{r}^{(k, i)}$.)

This step is carried out as follows: The input geometry in Cartesian coordinates $\mathbf{x}$ is transformed to internal coordinates $\mathbf{r}$ using the full nonlinear expressions that define the internal coordinates in terms of the Cartesians. The internal displacement coordinates are then calculated by eq 19. We then construct Taylor series expansions around each data point $(k, i)$ by combining $\Delta \mathbf{r}^{(k, i)}$ with the constants $D^{(k, i)}, \mathbf{b}^{(k, i)}$, and $\mathbf{C}^{(k, i)}$ calculated in step (vi), as in eq. 18 of ref 24 . Note that substituting eqs 20-23 of ref 24 into eq 18 of ref 24 yields eq 13 of ref 24 . Then, as in ref 24 but taking into account all symmetrically equivalent data points, we calculate the generalized distances and weights, carry out Shepard interpolation for $V_{12}$ as in eq 14 of ref 24 but with $m ! N$ terms in the sum:

$$
V_{12}^{S}(\mathbf{r})=\sum_{k=1}^{N} \sum_{i=1}^{m !} W_{k i}(\mathbf{r}) V_{12}^{\prime}(\mathbf{r}, k, i),
$$

where $W_{k i}$ are normalized weights discussed below (see also Supporting Information), and $V_{12}^{\prime}$ is defined by

$$
V_{12}^{\prime}(\mathbf{r}, k, i)=\sqrt{V_{12}(\mathbf{r}, k, i)^{2} u(\mathbf{r}, k, i)}
$$


where $V_{12}(\mathbf{r}, k, i)^{2}$ is given in eq 23 , and

$$
u(\mathbf{r}, k, i)=\left\{\begin{array}{cc}
\exp \left(-\delta / V_{12}(\mathbf{r}, k, i)^{2}\right) ; & V_{12}(\mathbf{r}, k, i)^{2}>0 \\
0 ; & \text { otherwise }
\end{array}\right.
$$

The derivatives of $V_{12}^{S}$ of eq 24, are calculated as in eqs 24-29 and 36-41 of ref 24 but summing over $k$ and $i$, not just $k$. These derivatives are given in the Supporting Information.

(viii) The derivatives of $V_{12}^{S}$ are then transformed from the internal coordinates to Cartesian coordinates by using the transformation matrices saved in step (vii), in the same fashion as in the formalism ${ }^{24}$ for nonsymmetrized potential energy surfaces.

(ix) Define matrix $\mathbf{V}$ at the input geometry $\mathbf{x}$ by

$$
\mathbf{V}(\mathbf{x})=\left(\begin{array}{cc}
\tilde{V}_{n}(\mathbf{x}) & V_{12}^{S}(\mathbf{x}) \\
V_{12}^{S}(\mathbf{x}) & \tilde{V}_{n}(\mathbf{x})
\end{array}\right)
$$

The lowest-energy eigenvalue of this matrix is the MCMM potential energy function. The diagonal matrix elements $\tilde{V}_{n}(\mathbf{x})$ and their derivatives $\tilde{\mathbf{G}}_{n}(\mathbf{x})$, and $\tilde{\mathbf{F}}_{n}(\mathbf{x})$ are obtained as follows: First we define

$$
\begin{aligned}
V_{\mathrm{MM}, n}^{(j)} & \equiv V_{n n}^{(j)}(\mathbf{x}) & j & =1, \ldots, m ! \\
\mathbf{G}_{\mathrm{MM}, n}^{(j)} & \equiv \frac{\partial}{\partial \mathbf{x}} V_{n n}^{(j)} & j & =1, \ldots, m !
\end{aligned}
$$

and

$$
\mathbf{F}_{\mathrm{MM}, n}^{(j)} \equiv \frac{\partial^{2}}{\partial \mathbf{x}^{2}} V_{n n}^{(j)} \quad j=1, \ldots, m !
$$

where, as before, each value of $j$ corresponds to one of the $m$ ! connectivity patterns. Then, $\tilde{V}_{n}(\mathbf{x}), \tilde{\mathbf{G}}_{n}(\mathbf{x})$, and $\tilde{\mathbf{F}}_{n}(\mathbf{x})$ are calculated as:

$$
\begin{gathered}
\tilde{V}_{n}(\mathbf{x})=-\Delta \ln \left(\frac{1}{\sigma_{n}} \sum_{j}^{m !} e^{-V_{\mathrm{MM}, n}^{(j)}(\mathbf{x}) / \Delta}\right) \\
\tilde{\mathbf{G}}_{n}(\mathbf{x})=\frac{\sum_{j}^{m !} \mathbf{G}_{\mathrm{MM}, n}^{j}(\mathbf{x}) e^{-V_{\mathrm{MM}, n}^{(j)}(\mathbf{x}) / \Delta}}{\sum_{j}^{m !} e^{-V_{\mathrm{MM}, n}^{(j)}(\mathbf{x}) / \Delta}}
\end{gathered}
$$


and

$$
\begin{aligned}
\tilde{\mathbf{F}}_{n}(\mathbf{x})= & \frac{\sum_{j}^{m !}\left(\mathbf{F}_{\mathrm{MM}, n}^{(j)}(\mathbf{x})-(1 / \Delta) \mathbf{G}_{\mathrm{MM}, n}^{(j)}(\mathbf{x}) \mathbf{G}_{\mathrm{MM}, n}^{(j)}{ }^{\mathrm{T}}(\mathbf{x})\right) e^{-V_{n}^{(j)}(\mathbf{x}) / \Delta}}{\sum_{j}^{m !} e^{-V_{\mathrm{MM}, n}^{(j)}(\mathbf{x}) / \Delta}}+ \\
& (1 / \Delta) \tilde{\mathbf{G}}_{n} \tilde{\mathbf{G}}_{n}^{\top} \sum_{j}^{m !} e^{-V_{\mathrm{MM}, n}^{(j)}(\mathbf{x}) / \Delta},
\end{aligned}
$$

where $V_{\mathrm{MM}, n}^{(j)}(\mathbf{x}), \mathbf{G}_{\mathrm{MM}, n}^{(j)}(\mathbf{x})$, and $\mathbf{F}_{\mathrm{MM}, n}^{(j)}(\mathbf{x})$ are sets of $m$ ! MM energies, gradients, and Hessians at the geometry $\mathbf{x}$.

(x) Find the eigenvalue $V$ of eq 27 and its derivatives in Cartesian coordinates. The lowest eigenvalue of eq 27 is given by

$$
V(\mathbf{x})=\frac{1}{2}\left(\tilde{V}_{1}(\mathbf{x})+\tilde{V}_{2}(\mathbf{x})\right)-\left[\left(\tilde{V}_{1}(\mathbf{x})-\tilde{V}_{2}(\mathbf{x})\right)^{2}+4\left(V_{12}^{S}(\mathbf{x})\right)^{2}\right]^{1 / 2}
$$

where $\tilde{V}_{n}$ are the symmetrized uninterpolated MM potentials given by eq 31 , and $V_{12}^{S}$ is the resonance integral obtained via the $m$ !N-term Shepard interpolation, eq 24 . The gradient and Hessian components of $V$ with respect to Cartesian coordinates are given by:

$$
G_{i}=\frac{\partial V}{\partial x_{i}}=\frac{1}{2}\left(\tilde{G}_{1 i}+\tilde{G}_{2 i}-\left(\frac{4 V_{12}^{S}\left(\frac{\partial V_{12}^{S}}{\partial x_{i}}\right)+\left(\tilde{V}_{1}-\tilde{V}_{2}\right)\left(\tilde{G}_{1 i}-\tilde{G}_{2 i}\right)}{\left(\left(\tilde{V}_{1}-\tilde{V}_{2}\right)^{2}+4\left(V_{12}^{S}\right)^{2}\right)^{1 / 2}}\right)\right)
$$

and

$$
\begin{aligned}
F_{i j} & =\frac{\partial^{2} V}{\partial x_{i} \partial x_{j}}=\frac{1}{2}\left(\tilde{F}_{1 i j}+\tilde{F}_{2 i j}\right. \\
& +\frac{\left(4 V_{12}^{S}\left(\frac{\partial V_{12}^{S}}{\partial x_{i}}\right)+\left(\tilde{V}_{1}-\tilde{V}_{2}\right)\left(\tilde{G}_{1 i}-\tilde{G}_{2 i}\right)\right)\left(4 V_{12}^{S}\left(\frac{\partial V_{12}^{S}}{\partial x_{i}}\right)+\left(\tilde{V}_{1}-\tilde{V}_{2}\right)\left(\tilde{G}_{1 j}-\tilde{G}_{2 j}\right)\right)}{\left(\left(\tilde{V}_{1}-\tilde{V}_{2}\right)^{2}+4\left(V_{12}^{S}\right)^{2}\right)^{3 / 2}} \\
& \left.-\frac{4\left(\frac{\partial V_{12}^{S}}{\partial x_{i}}\right)\left(\frac{\partial V_{12}^{S}}{\partial x_{j}}\right)+\left(\tilde{G}_{1 i}-\tilde{G}_{2 i}\right)\left(\tilde{G}_{1 j}-\tilde{G}_{2 j}\right)}{\left(\left(\tilde{V}_{1}-\tilde{V}_{2}\right)^{2}+4\left(V_{12}^{S}\right)^{2}\right)^{1 / 2}}-\frac{4 V_{12}^{S}\left(\frac{\partial^{2} V_{12}^{S}}{\partial x_{i} \partial x_{j}}\right)+\left(\tilde{V}_{1}-\tilde{V}_{2}\right)\left(\tilde{F}_{1 i j}-\tilde{F}_{2 i j}\right)}{\left(\left(\tilde{V}_{1}-\tilde{V}_{2}\right)^{2}+4\left(V_{12}^{S}\right)^{2}\right)^{1 / 2}}\right) .
\end{aligned}
$$


Note that steps (i)-(vi) are performed once at the beginning. Then steps (vii)-(x) are carried out every time that the dynamics algorithm needs the energy, gradient, and/or Hessian.

In the present article, the weight function $w(\mathbf{s})$ is evaluated as in eq 34 of ref 24 , but taking into account all symmetrically equivalent data points. For doing so, we first transform the Cartesian coordinates of the data points $(k, i)$ and the Cartesian coordinates of a geometry $\mathbf{x}$ (where one needs to evaluate the potential) to the set of the internal coordinates $\mathbf{s}$. The unnormalized weights are then calculated as

$$
w_{k i}(\mathbf{s})=\frac{\frac{1}{d_{k i}(\mathbf{s})^{4}}}{\sum_{k=1}^{(N+2)} \sum_{i=1}^{m !} \frac{1}{d_{k i}(\mathbf{s})^{4}}}
$$

where $d_{k i}$ is the generalized distance between $\mathbf{s}$ and $\mathbf{s}^{(k, i)}$ defined as:

$$
d_{k i}(\mathbf{s})=\sqrt{\sum_{\gamma=1}^{\Gamma m !}\left(s_{\gamma}-s_{\gamma}^{(k, i)}\right)^{2}},
$$

where $\mathbf{s} \equiv\left\{s_{1}, s_{2}, \ldots s_{\gamma}, \ldots s_{\Gamma}\right\}$. Note that the sum in eq 37 runs over $m !(N+2)$ data points where the $2 m$ ! extra data points correspond to the permutationally equivalent MM minima for the valence bond configurations $n=1$ and $n=2$. By way of contrast, the sum in eq 24 includes only $N m$ ! terms because we set $V_{12}$ equal to zero at MM minima because we assume that the potential near these points is well described by molecular mechanics. The omission of the extra $2 m$ ! terms in eq 24 combined with their inclusion in the denominator of eq 37 is equivalent to setting $V_{12}^{S}$ and its gradient and Hessian equal to zero at the MM minima.

For the present symmetrized application, $\Gamma=6$ because a four-body system has six internuclear distances. (This value is just accidentally the same as $m$ ! in the present case.) In most of the previous applications, ${ }^{24-29}$ we used $\Gamma=3$. The present article also includes, for comparison, some unsymmetrized calculations, and for those calculations we used $\Gamma=2$. 


\section{Application and Discussion of Results}

The two MM configurations used to represent asymptotic regions are $\mathrm{H}_{2} \mathrm{O}+\mathrm{H}(\mathrm{I})$ and $\mathrm{OH}+$ $\mathrm{H}_{2}$ (II). The MM parameters ${ }^{5,29,62,63}$ are given in Table 1 (note that there are no standard MM3 parameters for radicals).

For the present application, we used 3 training geometries. In particular, we place QM energies, gradients, and Hessians (a) at geometry of the saddle point, $\mathrm{HOHH}^{\neq}$, of reaction 1 (SP-I), (b) at the saddle point (SP-II, denoted $\mathrm{H}_{2} \mathrm{OH}^{\neq}$in eq 39) of the exchange reaction:

$$
\mathrm{H}_{2} \mathrm{O}+\mathrm{H} \rightarrow \mathrm{H}_{2} \mathrm{OH}^{\neq} \rightarrow \mathrm{OH}_{3} \rightarrow \mathrm{H}_{2} \mathrm{OH}^{\neq} \rightarrow \mathrm{H}_{2} \mathrm{O}+\mathrm{H}
$$

and (c) at the minimum (Min-I) that corresponds to an ammonia-like radical $\mathrm{OH}_{3}$. The geometries of these three Shepard points are given in Table 2. We will denote the potential constructed using three QM Hessians as MCMM(3). Fifteen other QM Hessians are obtained by symmetry without additional electronic structure calculations and are also used in interpolation. The parameter $\Delta$ was set equal to $0.04 E_{h}\left(1 E_{h}=1\right.$ hartree).

For the electronic structure calculations we use the hybrid density functional MPWB1K ${ }^{64}$ in conjunction with the $6-31+\mathrm{G}(\mathrm{d}, \mathrm{p})^{65}$ basis set; the geometries listed in Table 2 correspond to optimized structures at this level. Even though MPWB1K/6-31+G(d,p) significantly overestimates the barrier height for the exchange reaction (specifically, it gives $29.6 \mathrm{kcal} / \mathrm{mol}$ for the zero-point-exclusive barrier of reaction 2 as compared to $\sim 21 \mathrm{kcal} / \mathrm{mol}$ for the best estimate $^{57}$ ), it is sufficient to demonstrate the symmetry properties of the fit. (One should use more sophisticated electronic structure levels to generate QM energies and derivatives when one carries out dynamics calculations.) Geometrically, the intermediate structure MinI is rather close to the saddle point for this reaction being separated from the reactant well by a small barrier of $0.7 \mathrm{kcal} / \mathrm{mol}$ in MPWB1K/6-31+G(d,p) calculations and $0.3-0.8 \mathrm{kcal} / \mathrm{mol}$ in higher-level ${ }^{57}$ calculations. The forward and reverse zero-point-exclusive barriers for the abstraction reaction (1) are 5.0 and $18.6 \mathrm{kcal} / \mathrm{mol}$ with $\mathrm{MPWB} 1 \mathrm{~K} / 6-31+\mathrm{G}(\mathrm{d}, \mathrm{p})$ (cf. the best estimates ${ }^{66}$ of $5.1 \mathrm{kcal} / \mathrm{mol}$ and $21.2 \mathrm{kcal} / \mathrm{mol}$, respectively). 
Figure 2 shows two-dimensional cuts through the six-dimensional PESs as functions of the two symmetrically equivalent $r(\mathrm{OH})$ distances. The minimum corresponds to the $\mathrm{C}_{3 v}$ ammonia-like structure $\mathrm{OH}_{3}$ (Min-I). The top and bottom panels display the symmetrized PES and a nonsymmetrized PES, respectively. The nonsymmetrized PES was calculated with $N=2$ (QM Hessians are only placed at the saddle point of the $O-H_{a}$ dissociation channel and at the Min-I geometry) and with $\Gamma=3\left(\left\{O-H_{a} ; O-H_{b} ; O-H_{c}\right\}\right)$. This nonsymmetrized surface is called $\operatorname{MCMM}(2)$. The set of coordinates $\mathbf{r}$ for $\operatorname{MCMM}(2)$ includes these three coordinates plus the three $\mathrm{H}-\mathrm{O}-\mathrm{H}$ angles. Figure 2 shows that the symmetrized PES has the same shape in the two dissociation valleys, whereas the nonsymmetrized PES does not.

The upper panels of Figure 3 illustrate the symmetrized $\operatorname{MCMM}(3)$ surface near the saddle point SP-I of the abstraction reaction. These plots are made for the atomic labeling illustrated by the SP-I structure shown in Figure 1 and also for the labeling with $\mathrm{H}_{a}$ and $\mathrm{H}_{b}$ permuted. As one can see from these plots, the interpolated PES is invariant with respect to the exchange of the coordinates of the two hydrogen atoms. This is contrasted with the case of a nonsymmetrized PES that is shown in the lower panels of this Figure. This nonsymmetrized PES is constructed from one QM Hessian and is called $\operatorname{MCMM}(1)$. As in previous MCMM applications, ${ }^{24-29}$ the $\mathbf{r}$ coordinates are the natural vibrational coordinates for Shepard interpolation, and the s coordinates are the set of two distances that undergo significant changes $\left(\left\{O-H_{b} ; H_{b}-H_{c}\right\}\right)$. The lower right panel of Figure 3 shows that the potential is qualitatively incorrect at geometries corresponding to the reaction channel with the interchanged $\mathrm{H}_{a}$ and $\mathrm{H}_{b}$.

It is essential to note that while the MCMM method was originally developed to describe a potential in a localized region of a reaction swath of a particular reaction channel, the new formalism presented above is designed to describe (semi)global PESs that are suitable for study multiple reaction channels. We used a minimum of three QM Hessians (MCMM(3)) for the symmetrized PES and only one or two "reaction-specific" QM Hessians to describe 
the reaction channel of interest using the nonsymmetrized MCMM formalism. The potential shown in the upper panels of Figures 2 and 3 corresponds to a single PES obtained using three QM Hessians, whereas the cuts shown in the lower panels of these figures correspond to the two "different" nonsymmetrized surfaces (one constructed using the QM Hessians at SP-II and Min-I (Figure 1), and the other using the QM Hessian at SP-I (Figure 2)).

\section{Further Discussion and Comments}

An accurate PES has been previously developed to describe both the abstraction and exchange reaction channels of reaction 1 by Collins et al.$^{56}$ who pioneered Shepard interpolation as a general scheme to construct (semi)global PESs using a finite number of ab initio energies, gradients, and Hessians. ${ }^{47}$ To generate a PES sufficiently accurate for quantum scattering calculations, these authors used Shepard interpolation of the Taylor expansions of the potential energy at 1000 geometries. Thus that interpolation used 333 times more electronic structure Hessians than the present one. Although the goal of the present work is different, a key point is to note that in the MCMM approach, where one interpolates the resonance integral $V_{12}$ and uses previously calibrated MM potentials (along with the user-supplied parameters in reactions where the reactants or products or both are radicals), one generally needs far less electronic structure input data points as compared to the methods where one directly interpolates the potential energy.

Because actual evaluations of the diagonal and off-diagonal elements of the matrix $\mathbf{V}$ and their derivatives are performed in internal rather than in Cartesian coordinates, the MCMM PESs are invariant under the operation of inversion, ${ }^{51}$ and the symmetrized PES for the $\mathrm{H}_{2} \mathrm{O}+\mathrm{H}$ system is thus invariant under the operations the complete nuclear permutationinversion (CNPI) group. ${ }^{51}$ The latter is the direct product of the CNP group and the inversion group and has a very large order when the number of identical atoms in a system is large. In practice, however, one is rarely interested in all possible reactions of the atoms comprising 
a reactive system, but rather one identifies a few low-energy reaction paths. This implies that only a few identical nuclei in a system need to be treated as symmetrically equivalent while the other nuclei of the same kind can be considered as distinguishable. (For example, one might treat several protons in the active site of an enzyme symmetrically, but hydrogens far from the reactive site are effectively distinguishable over normal time scales.) The new MCMM scheme can therefore be applied to medium and large systems (e.g., using the recently presented ${ }^{28}$ strategy for evaluation of the resonance integral $V_{12}$ via hybrid QM/MM calculations) just as one would apply the older nonsymmetrized MCMM formalism, provided that one uses an appropriate set of internal coordinates for coordinate sets $\mathbf{r}$ and $\mathbf{s}$ (see Section III) so that all nuclear centers that need to be considered as indistinguishable are treated symmetrically in Shepard interpolation and in the calculation of the weight function. While the MM evaluations of the energies and energy derivatives at each geometry are now performed $m$ ! times (corresponding to all possible connectivity patterns of the identical nuclei), no extra computational cost is required to generate symmetrically equivalent sets of the gradient vectors and Hessian matrices at Shepard points at the QM level (which is the most computationally demanding part of the MCMM method).

In a broader context, $\mathrm{MCMM}^{24,25,27-29,67-69}$ may be considered as a way to extend molecular mechanics to chemical reactions. There are many computational methods in the literature that are designed to do this in one way or another. ${ }^{26,61, ?-107}$ Some of these are closely related to MCMM, whereas others are quite different. Furthermore, these methods were introduced to accomplish a variety of different objectives. For example, the empirical valence bond (EVB) method of Warshel and Weiss ${ }^{30}$ was introduced to transfer potential energy surfaces between environments by adding solvent effects to the diagonal Hamiltonian matrix elements. Either MCMM or EVB can be applied to reactions with more than one reaction pathway by adding additional valence bond structures (e.g., one could use a Hamiltonian matrix of order seven with three $\mathrm{OH}+\mathrm{H}_{2}$ structures, three $\mathrm{H}_{2} \mathrm{O}+\mathrm{H}$ structures, and 
one $\mathrm{OH}_{3}$ structure). This has some advantages, but it also has disadvantages, mainly that it leads to a larger matrix to be diagonalized. This in turn means that one must approximate more than one off-diagonal matrix element and that one can no longer take advantage of the simple form of the solution of the $2 \times 2$ Hamiltonian used here for obtaining analytic gradients and Hessians.

\section{Concluding Remarks}

We have presented an algorithm for using multi-configurational molecular mechanics to fit potential energy surfaces in a way that is manifestly symmetric under the permutation of identical nuclei, while retaining the simple structure of a Hamiltonian matrix of order 2. The algorithm allows us to represent potential energy surfaces in symmetry-equivalent reaction valleys as well as the ridge regions connecting them, and it leads to convenient formulas for analytic gradients and Hessians. The new method was illustrated by an application to the reaction of $\mathrm{H}_{2} \mathrm{O}$ with $\mathrm{H}$, for which we obtain a qualitatively correct semiglobal potential energy surface.

Acknowledgment. This work was supported by the United States Department of Energy, Office of Basic Sciences, under grant no. DE - FG02 - 86ER13579.

Supporting Information Available: Derivatives of $V_{12}^{S}$ of eq 24 with respect to internal coordinates. This information is available free of charge via the Internet at http://pubs.acs.org. 


\section{References}

[1] Olson, W. K.; Flory, P. J. Biopolymers 1972, 11, 25.

[2] Allinger, N. C. J. Am. Chem. Soc. 1977, 99, 8127.

[3] Burkert, U.; Allinger, N. L. Molecular Mechanics; American Chemical Society: Washington, 1984.

[4] van Gunsteren, W. F.; Berendsen, H. J. C.; Geurtsen, R. G.; Zwinderman, H. R. J. Ann. NY Acad. Sci. 1986, 482, 287.

[5] Allinger, N. C.; Yuh, Y.H.; Lii, J.-H. J. Am. Chem. Soc. 1989, 111, 8551.

[6] Lii, J.-H.; Allinger, N. L. J. Am. Chem. Soc. 1989, 111, 8566.

[7] Lii, J.-H.; Allinger, N. L. J. Am. Chem. Soc. 1989, 111, 8576.

[8] Cummins, P. L.; Gready, J. E. Chem. Phys. Lett. 1990, $173,355$.

[9] Tzuruki, S.; Tanabe, K. J. Chem. Soc. Perkin Trans. 2 1991, 181.

[10] Casewit, C. J.; Colwell, K. S.; Rappe, A. K. J. Am. Chem. Soc. 1992, 114, 10035. Casewit, C. J.; Colwell, K. S.; Rappe, A. K. J. Am. Chem. Soc. 1992, 114, 10046.

[11] Hagler, A. T.; Ewig, C. S. Computer Phys. Commun. 1994, 84, 131.

[12] Pearlman, D. A.; Case, D. A.; Caldwell, J. W.; Ross, W. S.; Cheatham, T. E. III; DeBolt, S.; Ferguson, D.; Seibel, G.; Kollman, P. Computer Phys. Commun. 1995, 91, 1.

[13] Rappe, A. K.; Colwell, K. S.; Casewit, C. J. Inorg. Chem. 1993, 32, 3438. 
[14] Cornell, W. D.; Cieplak, P.; Bayly, C. I.; Gould, I. R.; Merz, K. M., Jr.; Ferguson, D. M.; Spellmeyer, D. C.; Fox, T.; Caldwell, J. W.; Kollman, P. A. J. Am. Chem. Soc. 1995, 117, 5179 .

[15] Norrby, P.-O.; Waernmark, K.; Aekermark, B.; Moberg, C. J. Comp. Chem. 1995, $16,620$.

[16] Jorgensen, W. L.; Maxwell, D. S.; Tirado-Rives, J. J. Am. Chem. Soc. 1996, 118, 11225.

[17] Halgren, T. A. J. Comp. Chem. 1996, 17, 490.

[18] MacKerell, A. D., Jr.; Bashford, D.; Bellott, M.; Dunbrack, R. L.; Evanseck, J. D.; Field, M. J.; Fischer, S.; Gao, J.; Guo, H.; Ha, S.; Joseph-McCarthy, D.; Kuchnir, L.; Kuczera, K.; Lau, F. T. K.; Mattos, C.; Michnick, S.; Ngo, T.; Nguyen, D. T.; Prodhom, B.; Reiher, W. E., III; Roux, B.; Schlenkrich, M.; Smith, J. C.; Stote, R.; Straub, J.; Watanabe, M.; Wiorkiewicz-Kuczera, J.; Yin, D.; Karplus, M. J. Phys. Chem. B 1998, 102, 3586.

[19] Ponder, J. W.; Case, D. A. Adv. Protein Chem. 2003, 66, 27.

[20] Patel, S.; Brooks, C. L. III Mol. Simulation 2006, 32, 231.

[21] MacKerrell, A. D. Jr. J. Comp. Chem. 2004, 25, 1584.

[22] Jorgensen, W. L.; Tirado-Rives, J. Proc. Natl. Acad. Sci. USA 2005, 102, 6665.

[23] Marques, H. M.; Cukrowski, I. Phys. Chem. Chem. Phys. 2003 5, 5499.

[24] Kim, Y.; Corchado, J. C.; Villa, J.; Xing, X., Truhlar, D. G. J. Chem. Phys. 2000, 112, 2718.

[25] Albu, T.V.; Corchado, J.C.; Truhlar, D.G. J. Phys. Chem. A 2001, 105, 8465. 
[26] Truhlar, D. G. J. Phys. Chem. A 2002, 106, 5048.

[27] Lin, H.; Pu, J.; Albu, T.V.; Truhlar, D.G. J. Phys. Chem. A 2004, 108, 4112.

[28] Lin, H.; Zhao, Y.; Tishchenko, O.; Truhlar, D.G., J. Chem. Theor. Comp., 2006, 2, 1237.

[29] Tishchenko, O.; Truhlar, D. G. J. Phys. Chem. A, 2006, 110, 13530.

[30] Warshel, A.; Weiss, R. M. J. Am. Chem. Soc. 1980, 102, 6218.

[31] Garrett, B. C.; Truhlar, D. G.; Wagner, A. F.; Dunning, T. H. Jr., J. Chem. Phys. 1983, $78,4400$.

[32] Bondi, D. K.; Connor, J. N. L.; Garrett, B. C.; Truhlar, D. G. J. Chem. Phys. 1983, $78,5981$.

[33] Garrett, B. C.; Abusalbi, N.; Kouri, D. J.; Truhlar, D. G. J. Chem. Phys. 1985, 83, 2252.

[34] Truhlar, D. G.; Brown, F. B.; Steckler, R., Isaacson, A. D. In The Theory of Chemical Reaction Dynamics; Clary, D. C., Ed.; NATO ASI Series C170; D. Reidel: Dordrecht, Nederlands, 1986; p 285.

[35] Truhlar, D. G.; M. S. Gordon, M. S. Science 1990, 249, 491.

[36] Fernandez-Ramos, A.; Truhlar, D. G. J. Chem. Phys. 2001, 114, 1491.

[37] Truhlar, D. G. Garrett, B. C. Acc. Chem. Res. 1980, 440, 13.

[38] Truhlar, D. G.; Isaacson, A. D.; Skodje, R. T.; Garrett, B. C. J. Phys. Chem. 1982 86, 2252.; 1983, 87, 4554(E).

[39] Pechukas, P. Ber. Bunssenges. Physik. Chem. 1982, 86, 372. 
[40] Truhlar, D. G.; Isaacson, A. D.; Garrett, B. C. In Theory of Chemical Reaction Dynamics; Baer, M., Ed.; CRC Press: Boca Raton, FL, 1985; Vol. 4, p. 65.

[41] Allison, T. C.; Truhlar, D. G. In Modern Methods for Multidimensional Dynamics Computations in Chemistry; Thompson, D. L., Ed.; World Scientific: Singapore, 1998; p. 618.

[42] Garrett, B. C.; Truhlar, D. G. In Theory and Applications of Computational Chemistry: The First Forty Years; Dykstra, C. E.; Frenking, G.; Kim, K.; Scuseria, G., Eds.; Elsevier: Amsterdam, 2005; p 67.

[43] Truhlar, D. G.; Muckerman, J. T. In Atom-Molecule Collision Theory: A Guide for the Experimentalist; Bernstein, R. B., Ed.; Plenum Press, New York, 1979; p 505.

[44] Raff, L. M.; Thompson, D. L. In Theory of Chemical Reaction Dynamics; Baer, M., Ed.; CRC Press: Boca Raton, FL, 1985; Vol. 3, p 1.

[45] Mayne, H. R. In Dynamics of Molecules and Chemical Reactions; Wyatt, R. E., Zhang, J. Z. H., Eds.; Marcel Dekker: New York, 1996; p 589.

[46] Schatz, G. C.; Horst, M. T.; Takayanagi, T. In Modern Methods for Multidimensional Dynamics Calculations in Chemistry; Thompson, D. C., Ed.; World Scientific: Singapore, 1998; p 1. Tully, J. C.; ibid.; p. 34. Stanton, R. V.; Miller, J. L.; Kollman, P. A.; ibid.; p. 355; Jumpter, B. G.; Tuzon, R. E.; Noid, D. W.; ibid.; p. 401.

[47] Collins, M. A. Theor. Chem. Acc. 2002, 108, 313.

[48] Huang, X.; Braams, B.; Bowman, J. M. J. Chem. Phys. 2005, 122, 44308.

[49] Rutherford, D. E. Substitutional Analysis; Edinburgh University Press, London, 1948.

[50] Hamermesh, M. Group Theory; Addison-Wesley: Reading, MA, 1962; pp. 8ff. 
[51] Bunker, P. R.; Jensen, P. Molecular Symmetry and Spectroscopy; NRC Reaserch Press: Ottawa, 1998.

[52] Ischtwan,J.; Collins, M. A.; Watanabe, N. Hirao, K. J. Chem. Phys. 1994, 100, 8080.

[53] Nguyen, K. A.; Rossi, I.; Truhlar, D. G. J. Phys. Chem. 1995, $103,5522$.

[54] Bettens, R. P. A.; Collins, M. A.; J. Chem. Phys. 1999, 111, 816.

[55] Taketsugu, T.; Watanabe, N.; Hirao, K. J. Chem. Phys. 1999, 111, 3410.

[56] Zhang, D. Z.; Collins, M. A.; Lee, Soo-Y. Science 2000, 290, 961.

[57] Yang, M.; Zhang, D. H.; Collins, M. A.; Lee, S.-Y. J. Chem. Phys. 2001, 115, 174.

[58] Ponder, J. W. TINKER-Version 4.2; Washington University: St. Louis, MO, 2004.

[59] Jackels, C. F.; Gu, Z.; Truhlar, D. G. J. Chem. Phys. 1995, 102, 3188.

[60] Chuang, Y. Y.; Truhlar, D. G. J. Phys. Chem A 1998, 102, 242.

[61] Chang, Y. T.; Miller, W. H. J. Phys. Chem. 1990, 94, 5884.

[62] Herzberg, G. Molecular Spectra and Molecular Structure. I. Spectra of Diatomic Molecules; Van Nostrand Reinhold Company: New York, 1950.

[63] Handbook of Chemistry and Physics, Lide, D. R., Ed.; 87th edition; CRC Press: New York, 2006; p. 9-84.

[64] Zhao, Y.; Truhlar, D. G. J. Phys. Chem. A 2004, 108, 6908.

[65] Hehre, W. J.; Radom, L.; Schleyer, P. v. R.; Pople, J. A. Ab Initio Molecular Orbital Theory; Wiley: New York, 1986.

[66] Zhao, Y.; Lynch, B. J.; Truhlar, D. G. J. Phys. Chem A 2004, 108, 2715. 
[67] Kim, K. H.; Kim, Y. Bull. Korean Chem. Soc. 2003, 24, 763.

[68] Kim, K. H.; Kim, Y. J. Chem. Phys. 2004, 120, 623.

[69] Kim, K. H.; Kim, Y. J. Phys. Chem. A 2006, 110, 600.

[70] Jonston, H. S.; Goldfinger, P. J. Chem. Phys. 1962, 3\%, 700.

[71] Jonston, H. S.; Parr, C. A. J. Amer. Chem. Soc. 1963, 85, 2544.

[72] Raff, L. M. J. Chem. Phys. 1964, 60, 2220.

[73] Burton, G. W.; Sims, L. B.; Wilson, J. C.; Fry, A. J. Amer. Chem. Soc. 1977, 99, 3371.

[74] Joseph, T.; Steckler, R.; Truhlar, D. G. J. Chem. Phys. 1987, 87, 7036.

[75] Hwang, J.-K.; King, G.; Creighton, S.; Warshel, A. J. Amer. Chem. Soc. 1988, 110, 5297.

[76] Chang, Y. T.; Minichino, C.; Miller, W. H. J. Chem. Phys. 1992, 96, 4341.

[77] Bernardi, F., Olivucci, M.; Robb, M. A. J. Am. Chem. Soc. 1992, 114, 1606.

[78] Warshel, A.; Åqvist, J. J. Chem. Rev. 1993, 93, 2523.

[79] Grochowski, P.; Lesyng, B.; Bala, P.; McCammon, J. A. Int. J. Quantum Chem. 1996, $60,1143$.

[80] Field, M. J. In Computational Approaches to Biochemical Reactivity; Náray-Szabó, G., Warshel, A., Eds.; Understanding Chemical Reactivity; Kluwer: Dordrecht, 1997; p. 125. 
[81] Åqvist, J. In Computational Approaches to Biochemical Reactivity; Náray-Szabó, G., Warshel, A., Eds.; Understanding Chemical Reactivity; Kluwer: Dordrecht, 1997; p. 341.

[82] Rappe, A. K.; Pietsch, M. A.; Wiser, D. C.; Hart, J. R.; Bormann, L. M.; Skiff, W. M. Mol. Eng. 1997, 7, 385.

[83] Bentzien, J.; Muller, R. P.; Florian, J.; Warshel, A. J. Phys. Chem. B 1998, 102, 2293.

[84] Schmitt, U. W.; Voth, G. A. J. Phys. Chem. B 1998, 102, 5547.

[85] Vuilleumier, R.; Borgis, D. Chem. Phys. Lett. 1998, 284, 71.

[86] Brenner, D. W. Physica Status Solidi B 2000, 217, 23.

[87] Mo, Y.; Gao, J. J. Comp. Chem. 2000, 21, 1458.

[88] Espinosa-Garcia, J. J. Phys. Chem. A 2001, 105, 8748.

[89] van Duin, A. C. T.; Dasgupta, S.; Lorant, F.; Goddard, W. A., III J. Phys. Chem. A 2001, 105, 9396.

[90] Warshel, A.; Parson, W. W. Quart. Rev. Biophys. 2001, 34, 563.

[91] Brewer, M. L.; Schmitt, U. W.; Voth, G. A. Biophys. J. 2001, 80, 1691.

[92] Hammes-Schiffer, S.; Billeter, S. R. Int. Rev. Phys. Chem. 2001, 20, 591.

[93] Brenner, D. W.; Shenderova, O. A.; Harrison, J. A. Stuart, S. J.; Ni, B.; Sinnot, S. B. J. Phys. Condensed Matter 2002, 14, 783.

[94] Ginalski, P.; Grochowski, P.; Lesyng, B.; Shugar, D. Int. J. Quantum. Chem. 2002, 90, 1129.

[95] Olsen, P. T.; Jensen, F. J. Chem. Phys. 2002, 118, 3523. 
[96] Jensen, F.; Norrby, P.-O. Theor. Chem. Acc. 2003, 109, 1.

[97] Bochenkova, A. K.; Suhm, M. A.; Granovsky, A. A.; Nemukhin, A. V. J. Chem. Phys. 2004, 120, 3732 .

[98] Nielson, K. D.; Van Duin, A. C. T.; Oxgaard, J.; Deng, W.-Q.; Goddard, W. A., III J. Phys. Chem. A 2005, 109, 493.

[99] Bochenkova, A. K.; Firsov, D. A.; Nemukhin, A. V. Chem. Phys. Lett. 2005, 405, 165.

[100] Brancato, G.; Tuckerman, M. E. J. Chem. Phys. 2005, 122, 224507.

[101] Shurki, A.; Crown, H. A. J. Phys. Chem. B 2005, 109, 23638.

[102] Voth, G. Acc. Chem. Res. 2006, 39, 143.

[103] Shurki, A. Theor. Chem. Acc. 2006, 116, 253.

[104] Chakraborty, A.; Zhao, Y.; Lin, H.; Truhlar, D. G. J. Chem. Phys. 2006, 124, 44315.

[105] Schlegel, H. B.; Sonnenberg, J. L. J. Chem. Thery Comput. 2006, 2, 905.

[106] Truhlar, D. G. J. Comp. Chem. 2007, 28, 73.

[107] Lin, H.; Truhlar, D. G. Theor. Chem. Acc., in press. 
Table 1: Force field parameters for reaction $1 .^{a}$

\begin{tabular}{|c|c|c|c|c|c|c|c|c|}
\hline & \multicolumn{5}{|c|}{ van der Waals parameters } & \multicolumn{3}{|c|}{ Morse parameters } \\
\hline & atomic $\mathrm{H}$ & $\mathrm{H}$ in $\mathrm{H}_{2}$ & $\mathrm{H}$ in $\mathrm{H}_{2} \mathrm{O}$ & $\mathrm{O}$ in $\mathrm{OH}$ & $\mathrm{O}$ in $\mathrm{H}_{2} \mathrm{O}$ & $\mathrm{H}-\mathrm{H}$ in $\mathrm{H}_{2}$ & $\mathrm{O}-\mathrm{H}$ in $\mathrm{OH}$ & $\mathrm{O}-\mathrm{H}$ in $\mathrm{H}_{2} \mathrm{O}$ \\
\hline$r_{m}, \AA$ & 1.32 & 1.20 & 1.32 & 1.62 & 1.82 & & & \\
\hline$\epsilon, \mathrm{kcal} / \mathrm{mol}$ & 0.008 & 0.016 & 0.008 & 0.059 & 0.036 & & & \\
\hline$r_{e}, \AA$ & & & & & & $0.7414^{b}$ & $0.9707^{b}$ & $0.9470^{b}$ \\
\hline$f, \operatorname{mdyn} / \AA$ & & & & & & $5.75^{c}$ & 6.5 & 7.45 \\
\hline$D_{e}, \mathrm{kcal} / \mathrm{mol}$ & & & & & & $104.2^{d}$ & $120.0^{e}$ & $150.0^{e}$ \\
\hline
\end{tabular}

\begin{abstract}
$\bar{a}$ The van der Waals energy was calculated using the modified ${ }^{29}$ Exp-6 potential with the values for $A, B$, and $C$ as the original $\mathrm{MM}^{5}$ parameterization and the value for $D$ of 0.01 for the symmetrized PES and 0.005 for nonsymmetrized PES. For the bond stretching terms we used the Morse potential. For the angle bending potential in water we used the same functional form and the same parameters as in the original MM3 force field (these parameters are not shown in this Table). ${ }^{b}$ ref $62 .{ }^{c}$ ref $63 .{ }^{d}$ For the bond stretching term in $\mathrm{H}_{2}, D_{e}$ was set to be equal to $D_{298}^{o}$ of ref $63 .{ }^{e}$ For $\mathrm{OH}$ stretches, the values used for $D_{e}$ are larger than the actual equilibrium dissociation energies, which does not cause a problem because in MCMM the energy of reaction is evaluated by electronic structure calculations, not by molecular mechanics.
\end{abstract}


Table 2: Three geometries used in Shepard interpolation. Bond distances are in $\AA$ and bond angles are in degree.

\begin{tabular}{lcccccccc}
\hline \hline Structure & $r\left(\mathrm{OH}_{a}\right)$ & $r\left(\mathrm{OH}_{b}\right)$ & $r\left(\mathrm{OH}_{c}\right)$ & $r\left(\mathrm{H}_{b} \mathrm{H}_{c}\right)$ & $\angle \mathrm{H}_{a} \mathrm{OH}_{b}$ & $\angle \mathrm{H}_{a} \mathrm{OH}_{c}$ & $\angle \mathrm{H}_{b} \mathrm{OH}_{c}$ & $\angle \mathrm{H}_{c} \mathrm{H}_{b} \mathrm{O}$ \\
\hline SP-I & 0.965 & 1.322 & & 0.824 & 99.7 & & & 164.7 \\
SP-II & 1.122 & 0.992 & 0.992 & & 102.3 & 102.3 & 103.4 & \\
Min-I & 1.017 & 1.017 & 1.017 & & 102.6 & 102.6 & 102.6 & \\
\hline \hline
\end{tabular}




\section{Figure Captions}

Figure 1. Atom labels for the unique data points (SP-I, SP-II, and Min-I) used in Shepard interpolation.

Figure 2. Cuts through interpolated PESs plotted as functions of the $\mathrm{OH}_{a}$ and $\mathrm{OH}_{b}$ distances showing the exchange reaction (eq 39) for the symmetrized PES (top) and nonsymmetrized PES (bottom). All remaining internal coordinates are fixed at their values at the Min-I structure (see Table 2). Contour labels are in kcal/mol. The zero of energy corresponds to the $\mathrm{OH}+\mathrm{H}_{2}$ asymptote. The symmetrized PES shown in the upper panel is constructed using 3 QM Hessians $(\operatorname{MCMM}(3))$ and the unsymmetrized PES shown in the lower panel is constructed using 2 QM Hessians $(\operatorname{MCMM}(2))$, as explained in Section 3.

Figure 3. Cuts through interpolated PESs as functions of the $\mathrm{OH}$ and $\mathrm{HH}$ distances showing the abstraction reaction channel 1; symmetrized PES (upper left and right panels) and nonsymmetrized PES (lower left and right panels). Contour labels are in $\mathrm{kcal} / \mathrm{mol}$. Contours on the lower right plot are shown in increment of $10 \mathrm{kcal} / \mathrm{mol}$ starting from -8 $\mathrm{kcal} / \mathrm{mol}$. The zero of energy corresponds to the $\mathrm{OH}+\mathrm{H}_{2}$ asymptote. The numbers in parenthesis indicate the number of QM Hessians. The cuts shown on the left and right sides of the lower panel correspond to a nonsymmetrized MCMM(1) PES. 
Figure 1

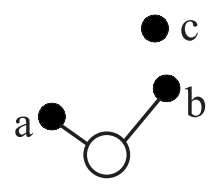

SP-I

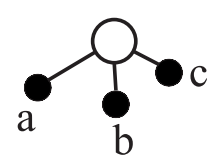

SP-II

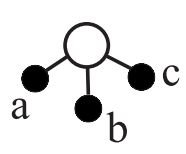

Min-I 
Figure 2
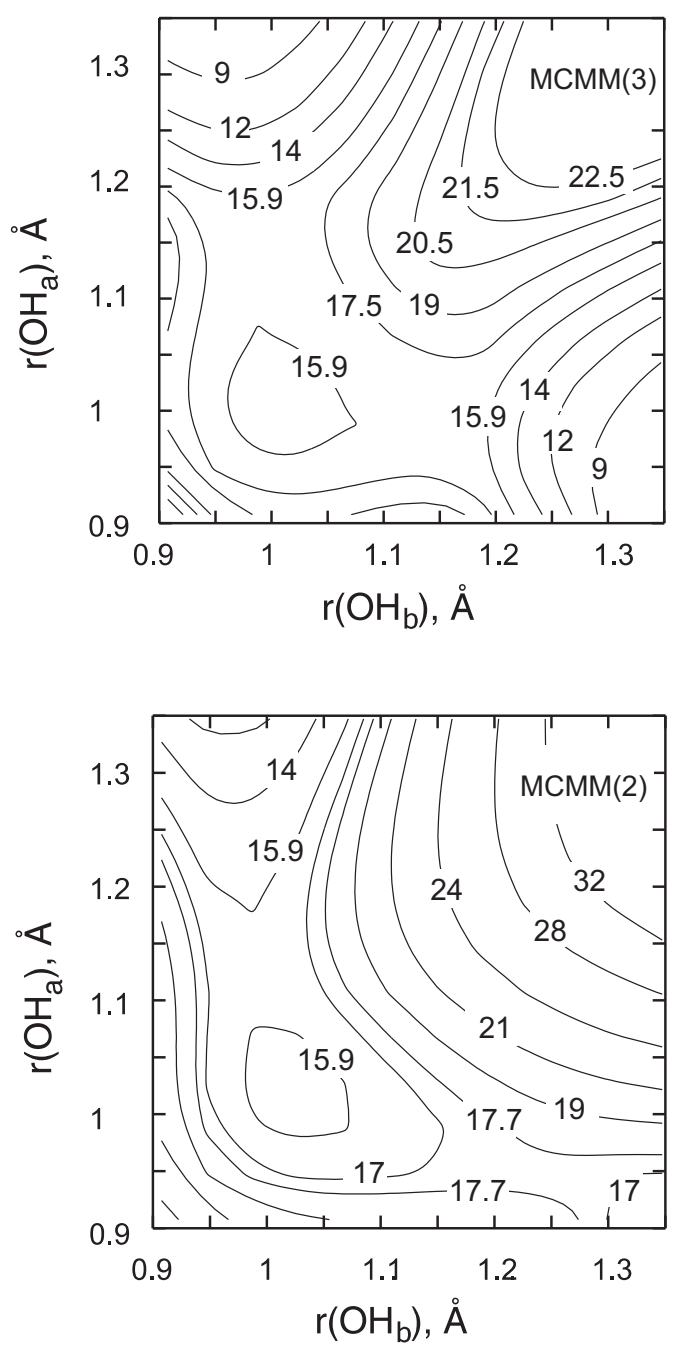
Figure 3
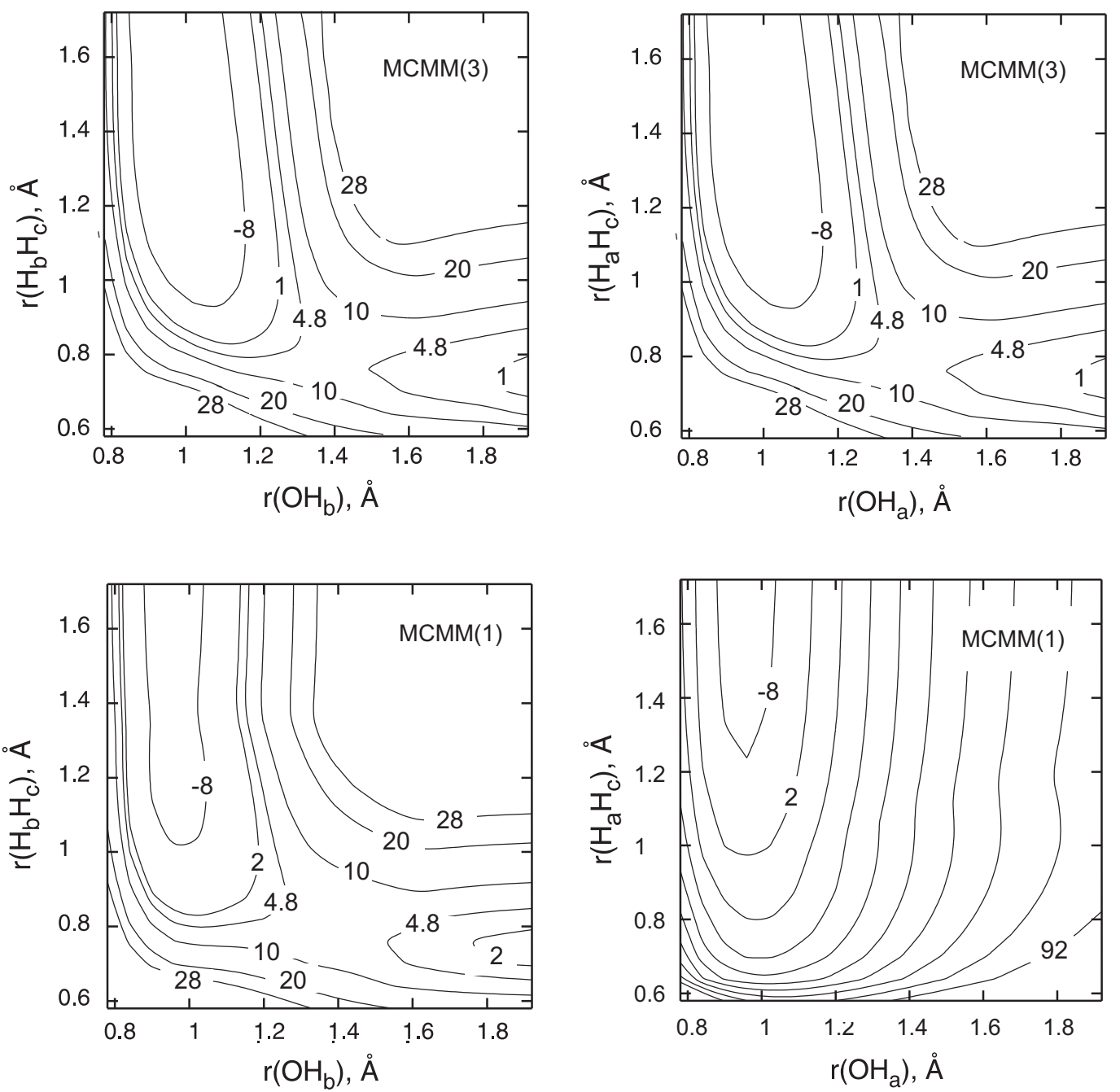